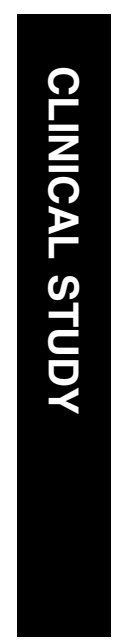

\section{Prevalence of myocilin gene mutations in a novel UK cohort of POAG patients}

${ }^{1}$ Genetic Epidemiology and Bioinformatics Group, Human Genetics Division (Mp 808), Southampton General Hospital SO16 6YD, University of Southampton, Southampton, UK

${ }^{2}$ Clinical Neurosciences Division (Mp 806), Southampton General HospitalSO166YD, University of Southampton, Southampton, UK

${ }^{3}$ Wessex Regional Genetics Laboratory, Salisbury District Hospital SP2 8BJ, Salisbury, UK

${ }^{4}$ Southampton Eye Unit, Southampton General Hospital SO16 6YD, Southampton, UK

Correspondence: A Lotery, Clinical Neurosciences Division, Mailpoint 806, Southampton General Hospital Tremona Raod, Southampton, SO16 6YD, UK

Tel: + 442380794590 ;

Fax: + 442380794542

E-mail: a.j.lotery@

southampton.ac.uk

Received: 9 August 2008 Accepted in revised form: 10 March 2009 Published online: 1 May 2009

\begin{abstract}
Purpose To identify the prevalence of myocilin gene mutations in a UK glaucoma cohort.

Methods Primary open-angle (POAG) and normal tension glaucoma patients were recruited from the Southampton University Hospital Trust Eye Clinic and satellite regional glaucoma clinics. Phenotype data relating to disease history and other potential risk factors were recorded and blood samples collected for each consenting participant. Point mutation analysis of the myocilin gene was carried out using six overlapping PCR fragments covering the entire coding sequence of the gene. A total of 316 POAG samples were examined of which $7(2.2 \%)$ tested positive for disease-causing mutations in this gene. One of these seven non-synonymous mutations represented a previously unreported amino-acid substitution of cysteine for arginine at codon 296 (p.R296C) of the myocilin protein. Conclusions This study identifies a $\mathbf{2 . 2 \%}$ prevalence of myocilin mutations in a cohort of ethnically homogenous glaucoma patients selected from a UK ophthalmic clinic. A novel myocilin mutation is also described. This study identifies that myocilin genetic screening is feasible in NHS glaucoma clinics for genetic counselling and cascade testing of relatives of patients affected by myocilin glaucoma.
\end{abstract}

Eye (2010) 24, 328-333; doi:10.1038/eye.2009.73; published online 1 May 2009

Keywords: glaucoma; POAG; cohort; myocilin

\section{Introduction}

Glaucoma is a term covering a heterogeneous group of conditions that have in common an
S Ennis', J Gibson', H Griffiths², D Bunyan³ AJ Cree', D Robinson ${ }^{3}$, J Self ${ }^{2,4}$, A MacLeod ${ }^{4}$ and A Lotery ${ }^{2,4}$ irreversible and usually progressive optic neuropathy resulting in distinctive patterns of visual field loss. Around $2 \%$ of the population have POAG beyond the age of 40 years, rising to around $4 \%$ at the age of 80 years. ${ }^{1}$ In the context of the ageing population, the prevalence of glaucoma will rise and become an even greater health problem. It is estimated that up to $35 \%$ of the retinal ganglion cells have already been lost before the visual field defects can be detected by perimetry, and subjective awareness of field loss usually occurs later still. ${ }^{2}$ Therefore, only after the eye has already suffered significant and permanent damage is a patient likely to be commenced on treatment. In developed countries, fewer than $50 \%$ of those with glaucoma are aware of their disease. ${ }^{3}$ There is currently no available screening method that detects glaucoma before permanent damage having occurred.

In the United Kingdom, the annual cost associated with blindness because of glaucoma probably exceeds $£ 100$ million. ${ }^{1}$ The real impact of the disease is almost certainly greater, as visual field loss can have a profound impact on the quality of life of the affected patient, such as loss of driving licence, even when visual acuity is relatively unaffected. In addition, treatments given for glaucoma, whether surgical or medical, can further reduce the patient's quality of life. On an individual basis, the lifestyle changes necessary when suffering from this chronic disease and the functional limitations resulting from loss of vision are immense. This loss of vision may be postponed or prevented by early diagnosis and appropriate treatment. However, with the current methods, early diagnosis still represents permanent visual loss. Once glaucoma is diagnosed, the patient has to be followed up by an ophthalmologist for the rest of his/her life. At present, between a 
quarter and a third of all patients attending ophthalmology clinics have glaucoma. This proportion, and the absolute number of patients, is set to increase as the population ages. First-degree relatives of individuals affected with glaucoma have up to an eightfold increased risk of developing glaucoma compared with the general population. ${ }^{4}$ First-degree relatives are, therefore, encouraged to have regular eye examinations, although only a fraction of these patients will ever develop glaucoma.

A major step forward in the understanding of POAG was the discovery that dominant mutations in the myocilin gene (MYOC; accession identifier NM_000261) are responsible for this condition in up to $4 \%$ of cases. ${ }^{5,6}$ Over 70 mutations have subsequently been identified by numerous other groups; $;^{7-10}$ however, this is a small fraction of the total number of patients affected with POAG. The extremely high prevalence of POAG in the general population means that this represents several tens of thousands of patients in the United Kingdom. The myocilin gene encodes the trabecular meshwork-induced glucocorticoid response (TIGR) protein and is expressed in the ciliary body, a tissue responsible for regulating aqueous humour secretion and intraocular pressure (IOP) ${ }^{11}$

As MYOC mutations are dominant and highly penetrant, there will, therefore, be a further cohort of first-degree relatives carrying high-risk MYOC alleles. Screening for MYOC mutations represents an opportunity to improve glaucoma management. It would allow patients known to carry MYOC mutations to be monitored closely in glaucoma clinics and to have treatment instituted at an earlier stage, thus preserving vision. This has already been shown to be effective in a small family study. ${ }^{12}$ As myocilin glaucoma is a dominant disease, family members of the index case could also be monitored and complications of glaucoma could be avoided, whereas family members who are negative for an MYOC mutation could be released from the follow-up. Currently, in ophthalmic practice in the United Kingdom, relatives of patients with POAG are screened in ophthalmic clinics or by opticians in the community. In either case, this can be costly for either the National Health Service (NHS) or for the patients themselves. Tailored screening is likely to provide financial savings in both cases by focussing resources on the highest risk groups. Furthermore, identifying groups with significantly greater risk of developing glaucoma may provide the stimulus for the development of less widely used screening modalities. Therefore, MYOC mutation screening would not significantly increase glaucoma services workloads, but rather concentrate resources on patients most at risk of developing myocilin glaucoma. Myocilin is an attractive gene to screen as the majority of mutations (93\%) are concentrated in one exon, namely exon 3 (http://www.myocilin.com/ variants.php), where the commonest mutation is $90 \%$ penetrant. ${ }^{12}$ MYOC screening fulfils the Wilson-Jungner criteria for appraising the validity of a screening programme. ${ }^{13}$ We evaluated how feasible it was to conduct myocilin glaucoma screening in a regional glaucoma service.

\section{Materials and methods}

Ethical approval for the collection of patient information and blood samples was provided by the Southampton and South West Hampshire Local Research Ethics Committee (05/Q1702/8) and Cohort Recruitment commenced in August 2005. The majority of study participants presented as outpatients at Southampton University Hospital Eye Unit, although ethical approval has recently been extended such that on-going recruitment can include patients presenting at smaller satellite glaucoma clinics within the region. Each patient was examined by an experienced glaucoma specialist. Diagnoses were made on the basis of characteristic visual field loss/glaucomatous optic disc damage/increased IOP. Patients presenting with narrow-angle, developmental or secondary glaucoma or any other known abnormalities of the anterior segment were excluded. Patients with unambiguous glaucoma, but normal tension were included in sample collection later. Furthermore, to select for patients with typical POAG or normal-tension glaucoma (NTG), only patients diagnosed over the age of 40 years were included. Both conditions are rare before this age.

Intra ocular pressure (IOP) data were taken at various time points and the maximum values for each eye (maxIOP) recorded for all recruits to allow stratification by disease subset during later analyses. Specialists also completed a patient information sheet for each participant detailing phenotypic and covariate data. This included date of diagnosis, treatment for hypertension, use of eye drops, incidence of myopia (any myopic correction) before 40 years of age, family history, ethnicity, and ocular surgery history. Participants were also asked about symptoms of Raynaud's syndrome. Ocular-specific data collected for each eye include IOP, vertical cup/disc ratio, presence of visual field defect, and corneal thickness. Quantitative data on the number of eye drops used, earlier trabeculectomy surgery, and history of optic disc haemorrhages were also collected.

DNA was extracted according to the standard methods, ${ }^{14}$ dissolved in TE buffer, and stored at $-20{ }^{\circ} \mathrm{C}$. DNA concentrations were measured using a NanoDrop 1000 spectrophotometer and normalised to $10 \mathrm{ng} / \mu \mathrm{l}$. In all, $15 \mu \mathrm{l}$ of DNA from 316 samples were plated into 
$4 \times 96$-well plates and dispatched on ice to the Wessex Regional Genetics Laboratory in Salisbury for MYOC gene screening. Also included on these plates were six duplicate samples and seven positive control samples, that is, individuals previously identified to carry MYOC gene mutations. These positive controls were provided by collaborators and did not form part of our cohort. The diagnostic technician was blind as to which wells contained the diagnostic controls.

Point mutation analysis of the myocilin gene was carried out using transgenomic wave dHPLC analysis of six PCR fragments, which cover the entire coding sequence of the gene and include two overlapping fragments for exon 1, one for exon 2, and three overlapping fragments for exon 3 (primer sequences and dHPLC conditions available on request). Any abnormal dHPLC results (suggestive of polymorphisms) were then followed up by direct sequencing of the relevant fragment using BigDye version 1.1 (Applied Biosystems, Foster City, USA, www.appliedbiosystems.com).

Statistical analyses were carried out using SPSS version 16.0 (SPSS Inc., Chicago, USA).

\section{Results}

Summary statistics are presented on a total of 316 unrelated Caucasian patients for whom DNA was available for study in Tables $1 \mathrm{a}$ and $\mathrm{b}$. The age range of the cohort was 40-98 years; mean age was 75 years, and median age was 77 years.

Table 1 Summary statistics of nominal (A) and quantitative (B) collected for 316 glaucoma patients

\begin{tabular}{|c|c|c|c|c|}
\hline (A) & $N$ & Yes $(\%)$ & No $(\%)$ & \\
\hline Family history & 303 & $138(45.5)$ & $165(54.5)$ & \\
\hline Raynaud's syndrome & 307 & $35(11.4)$ & $272(88.6)$ & \\
\hline Myopia < 40 years & 307 & $72(23.5)$ & $235(76.5)$ & \\
\hline Glaucoma visual field loss & 309 & $274(88.7)$ & $35(11.3)$ & \\
\hline Trabeculectomy & 306 & $108(35.3)$ & $198(64.7)$ & \\
\hline Haemorrhage & 308 & $31(10.1)$ & 277 (89.9) & \\
\hline (B) & $n$ & Minimum & Maximum & Mean \\
\hline $\begin{array}{l}\text { Maximum intraocular } \\
\text { pressure }^{\mathrm{a}}\end{array}$ & 303 & 14 & 50 & 26.27 \\
\hline Cup/disc ratio ${ }^{\mathrm{a}}$ & 311 & 0.1 & 1 & 0.74 \\
\hline Age at entering the study & 307 & 37 & 96 & 73.02 \\
\hline Age at diagnosis & 306 & 30 & 90 & 63.45 \\
\hline Duration of disease (years) & 306 & 0 & 40 & 9.51 \\
\hline
\end{tabular}

Frequency of various clinical factors for all patients where information was available (eg, of the 303 patients with data available, only $138(45.5 \%)$ report a positive family history).

The range and mean value for various quantitative measures were gathered.

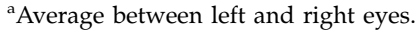

There was no significant gender difference in the sample ( $n=165$ men and 151 women; $P=0.43$ ). The mean IOP and cup/disc ratio measurements were 26.27 and $0.74 \mathrm{mmHg}$, respectively, although this did include 48 patients diagnosed with NTG. A positive family history was reported by $45.5 \%$ of the patients. A correlation matrix (Table 2), examining key diagnostic variables for high-tension and NTG groups separately, shows that cup/disc ratio and visual field loss are highly correlated. This correlation coefficient is very similar for both NTG and high-tension glaucoma, but the significance of this finding is most apparent in the larger sample of high-tension POAG patients. Within both the NTG and high-tension groups, patients tend to be diagnosed at an earlier age where there is a positive family history, but the strength of the significance is greater in the larger high-tension group $\left(P=2.04 \times 10^{-5}\right)$. An earlier age of diagnosis is also observed in patients designated myopic before the age of 40 years $\left(P=1.37 \times 10^{-4}\right)$. However, we cannot distinguish whether this is due to some 'double-hit' effect of having myopia and a predisposition for developing glaucoma or whether the younger age of diagnosis is simply due to an ascertainment bias introduced by regular optical checks in patients with myopia. Patients diagnosed at a later age tend to have more extensive visual field loss $(P=0.008)$. We speculate that as those with a positive family history are diagnosed at a younger age, this is likely to explain our observation that they have less extensive visual field loss $(P=0.005)$.

Mutation analysis correctly identified all seven diagnostic controls. All duplicate samples showed concordant results for the mutation screen. Of the 316 samples in our cohort, five patients carried the p.Q368x and one patient carried the p.T293K non-synonymous disease-causing mutations, both of which have been reported earlier in the MYOC gene (Table 3; http://www.myocilin.com/variants.php). One additional patient was shown to carry a novel point mutation causing a substitution of arginine for cysteine (Cys) at codon 296 (p.R296C) of the myocilin protein, and this unfavourable substitution was assumed to be disease causing because of the likely effect on the secondary structure of the protein. This mutation was not observed in any of the 630 chromosomes examined from the other 315 patients in our cohort. We observed no diseasecausing mutations within exons 1 or 2 of the gene, although many neutral polymorphisms were detected across all three exons of the gene, all of which had been reported earlier (http://www.myocilin.com/ variants.php). None of the seven patients had been previously aware of their MYOC mutation status and only four of these seven had indicated a family history of glaucoma. Interestingly, two of the seven patients had 
Table 2 Correlation matrix of key diagnostic variables for POAG (upper right diagonal) and NTG (lower left diagonal)

\begin{tabular}{|c|c|c|c|c|c|c|c|}
\hline & & $\mathrm{IOP}^{\dagger}$ & $\begin{array}{l}\text { Visual field } \\
\text { loss }^{\dagger}\end{array}$ & Cup:disc ratio ${ }^{\dagger}$ & $\begin{array}{c}\text { Age at } \\
\text { diagnosis }\end{array}$ & Myopia <40yrs & Family history \\
\hline $\mathrm{IOP}^{\dagger}$ & $\begin{array}{l}\text { Correlation Coefficient } \\
\text { Sig. (2-tailed) } \\
\mathrm{N}\end{array}$ & & $\begin{array}{c}-0.024 \\
0.701 \\
254\end{array}$ & $\begin{array}{c}-0.103 \\
0.100 \\
255\end{array}$ & $\begin{array}{c}-0.115 \\
0.069 \\
251\end{array}$ & $\begin{array}{c}-0.063 \\
0.319 \\
252\end{array}$ & $\begin{array}{c}-0.013 \\
0.835 \\
249\end{array}$ \\
\hline $\begin{array}{l}\text { Visual field } \\
\text { loss }^{\dagger}\end{array}$ & $\begin{array}{l}\text { Correlation Coefficient } \\
\text { Sig. (2-tailed) } \\
\mathrm{N}\end{array}$ & $\begin{array}{c}-0.010 \\
0.944 \\
48\end{array}$ & & $\begin{array}{c}0.403^{\star \star} \\
2.31 \mathrm{E}-11 \\
254\end{array}$ & $\begin{array}{c}0.168^{\star \star} \\
0.008 \\
250\end{array}$ & $\begin{array}{c}0.020 \\
0.748 \\
251\end{array}$ & $\begin{array}{c}-0.179^{* *} \\
0.005 \\
248\end{array}$ \\
\hline Cup:disc ratio ${ }^{\dagger}$ & $\begin{array}{l}\text { Correlation Coefficient } \\
\text { Sig. (2-tailed) } \\
\mathrm{N}\end{array}$ & $\begin{array}{c}-0.094 \\
0.524 \\
48\end{array}$ & $\begin{array}{l}0.479^{\star *} \\
0.001 \\
48\end{array}$ & & $\begin{array}{c}0.009 \\
0.887 \\
251\end{array}$ & $\begin{array}{c}-0.030 \\
0.639 \\
252\end{array}$ & $\begin{array}{c}-0.137^{\star} \\
0.031 \\
249\end{array}$ \\
\hline $\begin{array}{c}\text { Age at } \\
\text { diagnosis }\end{array}$ & $\begin{array}{l}\text { Correlation Coefficient } \\
\text { Sig. (2-tailed) } \\
\mathrm{N}\end{array}$ & $\begin{array}{c}-0.057 \\
0.709 \\
46\end{array}$ & $\begin{array}{c}-0.003 \\
0.985 \\
46\end{array}$ & $\begin{array}{c}0.054 \\
0.720 \\
46\end{array}$ & & $\begin{array}{c}-0.240^{* *} \\
1.37 E-04 \\
248\end{array}$ & $\begin{array}{c}-0.269^{* *} \\
2.04 E-05 \\
245\end{array}$ \\
\hline Myopia <40yrs & $\begin{array}{l}\text { Correlation Coefficient } \\
\text { Sig. (2-tailed) } \\
\mathrm{N}\end{array}$ & $\begin{array}{c}-0.058 \\
0.695 \\
48\end{array}$ & $\begin{array}{c}-0.229 \\
0.117 \\
48\end{array}$ & $\begin{array}{c}-0.166 \\
0.259 \\
48\end{array}$ & $\begin{array}{c}0.193 \\
0.198 \\
46\end{array}$ & & $\begin{array}{c}0.049 \\
0.444 \\
247\end{array}$ \\
\hline Family history & $\begin{array}{l}\text { Correlation Coefficient } \\
\text { Sig. (2-tailed) } \\
\mathrm{N}\end{array}$ & $\begin{array}{c}0.137 \\
0.359 \\
47\end{array}$ & $\begin{array}{c}0.015 \\
0.922 \\
47\end{array}$ & $\begin{array}{c}-0.080 \\
0.594 \\
47\end{array}$ & $\begin{array}{c}-0.340^{\star} \\
0.022 \\
45\end{array}$ & $\begin{array}{c}-0.025 \\
0.868 \\
47\end{array}$ & \\
\hline \multicolumn{3}{|c|}{$\begin{array}{l}* \text { *. Correlation is significant at the } 0.01 \text { level (2-tailed). } \\
* \text { Correlation is significant at the } 0.05 \text { level ( } 2 \text {-tailed). }\end{array}$} & $\begin{array}{l}\text {-TTG }(n=255) \\
\text { VTG }(n=48)\end{array}$ & & ean betv & e left and rig & \\
\hline
\end{tabular}

Data in the upper right segment represent those for high-tension glaucoma, whereas those in the bottom left segment are for the smaller group of normaltension glaucoma (NTG). Where data were collected for both eyes, for example, maximum intraocular pressure (maxIOP), the average between the left and right eye has been used for the analysis. For correlating any two outcomes, three statistical values are given: the first is the correlation coefficient, a value between -1 and +1 , which describes the direction and degree of the correlation; the second is the $P$-value showing the statistical strength of the observed correlation (highly significant findings $(P<0.01)$ are labelled ** and have been given a black background, marginally significant findings $(0.01<P<0.05)$ are labelled with a single asterisk *); the third value shows the number of patients (who had data available on both correlates) upon which the results are based. For example, the most striking result shows highly significant $\left(P=2.3 \times 10^{-11}\right)$ correlation between those with visual field loss and elevated cup/disc ratio - for the high-tension glaucoma patients, these results are based on 254 observations.

Table 3 Seven cases carrying a disease-causing MYOC mutation.

\begin{tabular}{llcr}
\hline Mutation & IOP & Age at diagnosis & Clinical diagnosis \\
\hline p.Q368X & 22.5 & 63 & POAG \\
p.Q368X & 42.5 & 50 & POAG \\
p.Q368X & 21 & 57 & NTG \\
p.Q368X & 30 & 43 & POAG \\
p.Q368X & 20.5 & 76 & NTG \\
p.T293K & 24 & 71 & POAG \\
p.R296C & 27 & 45 & POAG \\
Group & Mean IOP $(\mathrm{n})$ & Mean age at diagnosis $(\mathrm{n})$ & $57.9(7)$ \\
Those with $M Y O C$ mutation & $26.8(7)$ & $63.6(299)$ & \\
Those without $M Y O C$ mutation & $26.3(296)$ &
\end{tabular}

$\mathrm{IOP}=$ intraocular pressure; NTG = normal-tension glaucoma, POAG = primary open-angle glaucoma.

Brief listing of the maximum observed IOP and age at diagnosis for each patient found to carry a disease-causing $M Y O C$ mutation. The two lines at the bottom show the mean values for these two variables averaged across those seven patients carrying an MYOC mutation vs those patients (with IOP and age at diagnosis data available) who did not carry a mutation.

a clinical diagnosis of NTG. All four patients reporting a positive family history were carriers of the p.Q368X mutation.

Some reports have suggested glaucoma patients carrying MYOC disease-causing mutations present at an earlier age and/or with IOP greater than that in non-MYOC glaucoma. ${ }^{15}$ We examined IOP and age at onset in these seven patients compared with the rest of the sample, and found no evidence for a younger age at onset $(P=0.21)$ or significantly higher IOP $(P=0.81)$. However, this may not be surprising given five of our patients carried the p.Q368X mutation that one study identified as not conferring such a predisposition to early onset or unusually high IOP. ${ }^{16}$ 


\section{Discussion}

This study highlights the feasibility of detecting myocilin glaucoma mutations in a regional NHS glaucoma service. In this clinic, a prevalence rate of $2.2 \%$ was detected. If the age of inclusion of patients had been widened, for example, to include juvenile glaucoma, the prevalence is likely to have been higher. Positive control DNA used for this work was ascertained through previous diagnostic assay.

Dominant mutations in the myocilin gene represent the primary genetic cause for POAG identified to date and are responsible for up to $4 \%$ of cases in populations studied thus far. Using dHPLC analysis followed by direct sequencing, we have screened the entire coding region of this gene in our novel cohort and identified seven patients carrying disease-causing mutations. None of these patients had been previously aware of their MYOC gene mutation status and those who had indicated a preference to be informed of any clinically significant results (on their study consent forms) have subsequently been advised. As a direct result of this new information, a number of non-symptomatic relatives of these patients have now presented for myocilin screening.

Importantly, following on from the validation of this assay in this study, the Wessex Regional Genetics Laboratory now offers this test as part of the NHS, thus making this service available to clinicians throughout the United Kingdom. The cost to the NHS for each sample is approximately $£ 1$ per PCR fragment per patient (excluding cost of staff and overheads). Any relatives identified as carriers of a disease-causing MYOC mutation can be closely monitored for signs of glaucoma. Very early detection and treatment instigation may significantly ameliorate vision loss in these individuals. Furthermore, by identifying first-degree relatives without MYOC mutations, these patients may be released from the traditional intensive screening programmes. This would offer significant savings to both patients and the NHS.

Earlier studies of unrelated glaucoma patients observed approximately 2-4\% frequency of myocilin mutations in unrelated glaucoma patients from various ethnic groups ${ }^{17}$ (ie, $4.3 \%$ in patients from Iowa, USA; $2.6 \%$ in African Americans from New York ; $2.8 \%$ in Japanese patients; $3.0 \%$ in Canadian patients; and $2.8 \%$ in Australian patients). Our screen identifies an overall prevalence of $2.2 \%$ in Caucasian POAG /NTG patients ascertained through ophthalmic clinics in Southern England. Similar to other studies, the disease-causing mutations detected in this population tended to occur in exon 3. One observed mutation has not been reported earlier (R296C) and may represent a population-specific variant. The introduction of a Cys residue by this variant is likely to represent a pathogenic mutation. Relative to other amino acids in proteins, Cys residues are often functionally important. They play important roles in a variety of functions, including metal binding and formation of disulphide bonds that alter protein structure. ${ }^{18}$ For these reasons, mutations causing the appearance or loss of this residue are often pathogenic. Furthermore, the 296 location within the myocilin gene shows significant evolutionary conservation (UniProt database http:/ / www.uniprot.org/) through to the Zebrafish species Danio rerio (data not presented). This also suggests an important role for the wild-type arginine residue in this position.

Typically, both POAG and NTG occur after the age of 40 years. For this reason, patients who were diagnosed with either condition before this age were excluded from this study. Therefore, genetic mutations, which tend to cause early onset or childhood glaucoma (neither of which are the primary focus of this study) have been selected against to identify mutations causing 'typical' POAG/NTG.

Diagnosis of glaucoma can be extremely complex and identification of the precise age at which glaucomatous disease started can be extremely difficult. We have validated a genetic test for myocilin glaucoma, which is now available in the NHS in an accredited NHS Regional Genetics Laboratory. Now, it provides NHS clinicians with another tool to identify patients (and their family members) suffering from myocilin glaucoma. This should permit earlier diagnosis and consequently reduce the late stage complications of glaucoma.

\section{Acknowledgements}

We thank our collaborators Edwin Stone and John Fingert from the University of Iowa for providing positive controls for the MYOC screening. We thank the research nurses of the Wellcome Trust Clinical Research Facility who collected patient information and blood samples and we are very grateful to all patients who kindly agreed to partake in this study. This study was funded by the Gift of Sight Appeal, the International Glaucoma Association and the UK Eire Glaucoma Society.

\section{References}

1 Royal College of Ophthalmologists. Royal College of Ophthalmologists. A national research strategy for ophthalmology. RCOphth publication, March 2002. 
2 Kerrigan-Baumrind LA, Quigley HA, Pease ME, Kerrigan DF, Mitchell RS. Number of ganglion cells in glaucoma eyes compared with threshold visual field tests in the same persons. Invest Ophthalmol Vis Sci 2000; 41: 741-748.

3 Quigley HA. Number of people with glaucoma worldwide. Br J Ophthalmol 1996; 80: 389-393.

4 Johnson AT, Alward WLM, Sheffield VC, Stone EM. In: Ritch R, Shields MB, Krupin T (eds). The Glaucomas, 2nd ed. St Louis: Mosby, 1996.

5 Fingert JH, Heon E, Liebmann JM, Yamamoto T, Craig JE, Rait J et al. Analysis of myocilin mutations in 1703 glaucoma patients from five different populations. Hum Mol Genet 1999; 8: 899-905.

6 Stone EM, Fingert JH, Alward WL, Nguyen TD, Polansky JR, Sunden SL et al. Identification of a gene that causes primary open angle glaucoma. Science 1997; 275: 668-670.

7 Xie X, Zhou X, Qu X, Wen J, Tian Y, Zheng F. Two novel myocilin mutations in a Chinese family with primary open-angle glaucoma. Mol Vis 2008; 14: 1666-1672.

8 Wirtz MK, Konstas AG, Samples JR, Kaltsos K, Economou A, Dimopoulos A et al. Myocilin variations and familial glaucoma in Taxiarchis, a small Greek village. Mol Vis 2008; 14: 774-781.

9 Kanagavalli J, Pandaranayaka E, Krishnadas SR, Krishnaswamy S, Sundaresan P. A review of genetic and structural understanding of the role of myocilin in primary open angle glaucoma. Indian J Ophthalmol 2004; 52: 271-280.

10 Sripriya S, Uthra S, Sangeetha R, George RJ, Hemamalini A, Paul PG et al. Low frequency of myocilin mutations in
Indian primary open-angle glaucoma patients. Clin Genet 2004; 65: 333-337.

11 Escribano J, Ortego J, Coca-Prados M. Isolation and characterization of cell-specific cDNA clones from a subtractive library of the ocular ciliary body of a single normal human donor: transcription and synthesis of plasma proteins. J Biochem 1995; 118: 921-931.

12 Fingert JH, Stone EM, Sheffield VC, Alward WL. Myocilin glaucoma. Surv Ophthalmol 2002; 47: 547-561.

13 Brindle P, Fahey T. Primary prevention of coronary heart disease. Br Med J 2002; 325: 56-57.

14 Miller SA, Dykes DD, Polesky HF. A simple salting out procedure for extracting DNA from human nucleated cells. Nucleic Acids Res 1988; 16: 1215.

15 Hewitt AW, Bennett SL, Fingert JH, Cooper RL, Stone EM, Craig JE et al. The optic nerve head in myocilin glaucoma. Invest Ophthalmol Vis Sci 2007; 48: 238-243.

16 Graul TA, Kwon YH, Zimmerman MB, Kim CS, Sheffield $\mathrm{VC}$, Stone EM et al. A case-control comparison of the clinical characteristics of glaucoma and ocular hypertensive patients with and without the myocilin Gln368Stop mutation. Am J Ophthalmol 2002; 134: 884-890.

17 Fingert JH, Heon E, Liebmann JM, Yamamoto T, Craig JE, Rait J et al. Analysis of myocilin mutations in 1703 glaucoma patients from five different populations. Hum Mol Genet 1999; 8: 899-905.

18 Beeby M, O'Connor BD, Ryttersgaard C, Boutz DR, Perry LJ, Yeates TO. The genomics of disulfide bonding and protein stabilization in thermophiles. PloS Biol 2005; 3: e309. 